\title{
EDITORIAL
}

\section{0 volumes of Diseases of Aquatic Organisms}

\author{
Alex Hyatt ${ }^{1}$, Sven Klimpel ${ }^{2}$, John Austin ${ }^{3, *}$, Otto Kinne ${ }^{3}$ \\ ${ }^{1}$ CSIRO, Livestock Industries, Australian Animal Health Laboratory, Geelong, Victoria 3220, Australia \\ ${ }^{2}$ Biodiversity and Climate Research Centre (BiK-F), Johann Wolfgang Goethe-University, 60325 Frankfurt am Main, Germany \\ ${ }^{3}$ Inter-Research, Nordbünte 23, 21385 Oldendorf/Luhe, Germany
}

With the present issue, the 100th volume of Diseases of Aquatic Organisms (DAO) has been reached. After 27 years, DAO is established as a journal of quality and central importance to its field.

DAO was founded in 1985 by Prof. Dr. Dr. h.c. Otto Kinne, who, with the vision and dedication that brought many other journals to leading positions, managed it for over 2 decades. As Founding Editor and as Director of the Inter-Research Science Center, he still presides over the journal. Since 2008, Alex Hyatt and Sven Klimpel have headed the Editorial Board as Editors-in-Chief, while John Austin, Assistant Managing Editor since the first volume, has served as Managing Editor.

DAO has set standards in scientific publishing at all levels: in the review process, in production, and above all in personal and prompt attention to all correspondence with authors, editors and reviewers. Nonetheless, in providing these services to the scientific community, we do everything possible to keep prices down. Subscription fees are moderate and, at 700 Euros, the cost to authors of publishing their article with Open Access is low. The high quality of photo reproduction has always been among the hallmarks of the journal, and no charges are made for colour printing. Our policy of providing free access to archived articles older than 5 years is far from commonplace among commercial publishers and has been warmly welcomed by the community.

As stated in the journal's aims: 'Diseases affect all facets of life-at the cell, tissue, organ, individual, population and ecosystem level.' Disease is a key ecological phenomenon, and DAO operates at the juncture of medical/veterinary science and ecology. Aquaculture species have always been represented strongly in the journal's pages, but the range of organisms affected by disease (and thus the scope of the journal) covers the full taxonomic spectrum. No less wide is the range of causative agents, which include viruses, bacteria, fungi, diverse protozoan and metazoan parasites, and environmental as well as anthropogenic factors. In recent years the spread of chytridiomycosis in amphibians has been a focus of DAO articles. Special Issues have also been dedicated to topics as diverse as coral diseases, marine vertebrate zoonoses, and welfare of aquatic organisms.

Competition for space in DAO is greater than ever, resulting in rejection rates of over $50 \%$. Although we do not support the excessive emphasis sometimes given to the Impact Factor (IF), we can point with satisfaction to our consistently respectable IFs of above 1.5, with the latest figure rising to 2.2. DAO is included in the PubMed database, and all articles can be accessed via direct links from PubMed entries. DAO is used in many laboratories, and key researchers state that they regard DAO as the premium journal for disseminating information on applied topics and on diseases of aquatic wildlife.

The success of DAO rests on the high quality of contributions submitted, the high performance of our editors and reviewers, the hard work of our technical and administrative staff, and the loyalty of our subscribers and readers.

Thank you to all - we will be needing your combined support for the next 100 ! 